\title{
Comparación de costes entre la nefrectomía radical laparoscópica y la abierta en el adenocarcinoma renal
}

\author{
Herranz Amo F, Subirá Ríos D, Hernández Fernández C, Martínez Salamanca JI, \\ Monzó JI, Cabello Benavente R.
}

Servicio de Urología. Hospital General Universitario Gregorio Marañón. Madrid.

Actas Urol Esp. 2006;30(9):921-925

\section{RESUMEN}

COMPARACIÓN DE COSTES ENTRE LA NEFRECTOMÍA RADICAL LAPAROSCÓPICA Y LA ABIERTA EN EL ADENOCARCINOMA RENAL

Objetivo: Realizar una comparación de costes (minimización de costes) entre la nefrectomía laparoscópica y la nefrectomía "abierta" trasperitoneal en el tratamiento del adenocarcinoma de riñón.

Método: Estudio retrospectivo sobre los primeros 26 pacientes sometidos a nefrectomía laparoscópica sin complicaciones intra ni postoperatoria en el periodo 2002-2003, utilizando como control a 22 pacientes tratados con nefrectomía "abierta" con las mismas características y en el mismo periodo de tiempo. Se evaluaron variables demográficas (edad, sexo, índice de masa corporal, tamaño tumoral, etc.), intraoperatorias (tiempo de quirófano y material fungible utilizado) y postoperatorias (tiempo de estancia en reanimación, necesidades de analgesia y estancia hospitalaria). Se aplicaron los costes calculados para nuestro Hospital y los imputados al Servicio de Urología en el año 2003, así como el coste de fungibles con IVA para el mismo año. Se realizó una comparación de costes entre ambos grupos.

Resultados: No hubo diferencias entre las variables demográficas entre ambos grupos excepto en el tamaño tumoral, mayor en las nefrectomías abiertas ( $\mathrm{p}=0,001)$. La nefrectomía laparoscópica fue un 29,4\% globalmente más cara que la abierta. El coste intraoperatorio (quirófano, anestesia y fungibles) de la laparoscópica excedió en un 151,6\% al de la abierta, mientras que en la abierta el coste postoperatorio fue superior en un 63\% con respecto a la laparoscópica.

Conclusiones: La nefrectomía laparoscópica en nuestro Centro es más cara que la nefrectomía abierta debido a una mayor ocupación de quirófano y a que el material fungible específico utilizado en el acto quirúrgico tiene un coste muy elevado. Sería necesario la reducción de forma importante del tiempo quirúrgico y la disminución del gasto en fungibles para que la nefrectomía laparoscópica pudiera ser competitiva en nuestro Hospital.

Palabras claves: Riñón. Laparoscopia. Nefrectomía. Análisis de costes.

\section{ABSTRACT}

OPENED VS. LAPAROSCOPIC RADICAL NEPHRECTOMY IN RENAL ADENOCARCINOMA COST COMPARISON

Purpose: To undertake a cost comparison (cost minimization) between transperitoneal laparoscopic and opened nephrectomy in renal adenocarcinoma treatment.

Method: Retrospective study on the first 26 patients submitted to LN without intra or postoperative complications in the period 2002-2003, using as control 22 patients treated with ON with the same characteristics and in the same period. Demographic variables were evaluated (age, sex, tumor size, etc.), intraoperative (operative time and fungible material used) and postoperative (length of stay in Postanaesthesic Care Unit, Acute Pain Unit needs and hospital stay). Our Hospital costs plus those imputed during year 2003 to the Urology Service, as well as the cost of fungible material for the same year were applied, carrying out a comparison of costs between both groups.

Results: There were no differences between the demographic variables between both groups except in the tumor, bigger size in the opened nephrectomy $(\mathrm{p}=0,001)$. Transperitoneal laparoscopic was $29,4 \%$ globally more expensive than opened nephrectomy. The transperitoneal laparoscopic intraoperative cost (operating room, anesthesia and fungibles) the exceeded in $151,6 \%$ to that of the opened nephrectomy, whereas in the opened nephrectomy the postoperative cost was a $63 \%$ higher than in the transperitoneal laparoscopic cases.

Conclusions: Transperitoneal laparoscopic in our Center is more expensive than opened nephrectomy due to a major occupation of operating room and that the specific fungible material used at the surgical act has a very high cost. It would be necessary to drastically reduce surgical time and decrease fungible material expenses, thus transperitoneal laparoscopic procedure could be competitive in our Hospital. 
$\mathrm{L}$ a nefrectomía radical se considera la técnica estándar para el tratamiento del cáncer renal desde que Robson ${ }^{1}$, en 1969 comunicara el aumento de supervivencia al compararla con series históricas tratadas con nefrectomía simple.

La primera nefrectomía laparoscópica fue realizada por Clayman et $\mathrm{al}^{2}$ en 1990. Desde entonces se han establecido los beneficios de la nefrectomía laparoscópica en cuanto a: menor dolor postoperatorio, disminución de la morbilidad, menor estancia hospitalaria, recuperación más rápida y mejores efectos estéticos ${ }^{3}$. También es verdad que la nefrectomía laparoscópica precisa de una curva de aprendizaje más larga, se utiliza más tiempo de quirófano y el material fungible utilizado es más caro.

Aunque se han descrito implantes tumorales en los puntos de entrada de los trócares en la cirugía de otros tumores (digestivos y ginecológicos), en la cirugía laparoscópica del adenocarcinoma renal es un hecho excepcional ${ }^{4,5}$, sobre todo cuando no se fragmenta la pieza para su extracción. La supervivencia cáncer específica a los 5 años es similar en los pacientes tratados mediante nefrectomía laparoscópica o nefrectomía abierta ${ }^{3-6}$.

El objetivo de este estudio es realizar un análisis de costes (minimización de costes) entre los pacientes con cáncer renal tratados con nefrectomía radical laparoscópica o nefrectomía radical trasperitoneal.

\section{MATERIAL Y MÉTODO}

Se seleccionaron retrospectivamente los primeros 26 pacientes en los que la vía de abordaje fue laparoscópica sin que presentaran complicaciones quirúrgicas ni postoperatorias durante el periodo 2002-2003. Como grupo control hemos utilizado a 22 pacientes con tumores menores de $10 \mathrm{~cm}$ en los que la vía de abordaje fue trasperitoneal, en el mismo periodo de tiempo, y no tuvieron complicaciones quirúrgicas ni postoperatorias. Por lo tanto tenemos un grupo (casos) que incluye 26 pacientes tratados mediante nefrectomía laparoscópica (NL) y un grupo (controles) que incluye a 22 pacientes tratados con nefrectomía abierta (NA). El diagnóstico, el estudio de extensión y la valoración pre-anestésica se realizó de la misma forma para ambos grupos. En todos los pacientes se reservaron 3 unidades de sangre para la intervención quirúrgica.

Las variables a medir para ambos grupos las podemos clasificar en: 1) Demográficas: edad, sexo, lateralidad renal, indice de masa corporal $\left(\mathrm{IMC}=\right.$ peso en $\mathrm{Kg} /$ talla en $\mathrm{cm}^{2}$ ), riesgo anestésico según la American Society of Anesthesiologist (ASA) y tamaño tumoral; 2) Intraoperatorias: tiempo de utilización del quirófano (desde que entra hasta que sale el paciente), tiempo de cirugía (desde el momento de la incisión hasta el término de la sutura de piel) y material fungible utilizado; 3) Postoperatorias: tiempo en la Unidad de Reanimación Postoperatoria Aguda (URPA), necesidad y duración de analgesia suministrada por la Unidad del Dolor Agudo (UDA) y tiempo de estancia hospitalaria.

El coste de la hora de quirófano calculado para nuestro Hospital en el ejercicio 2003 fue de $169 €$. El coste por hora del Servicio de Anestesia fue de $127 €$. El coste de la URPA se ha estimado en $3 €$ la hora, siendo el de la UDA de $17 €$ día. El coste de la estancia hospitalaria imputado al Servicio de Urología ascendió a $298 €$ día. Todos los costes expuestos recogen los componentes directos, indirectos y estructurales (gerencia, servicios administrativos, seguridad, etc), conforme a la distribución comúnmente aceptada.

Aunque hubo ligeras variaciones en el material fungible utilizado en la NL, podemos decir que en cada NL se utilizaron: un reductor $(2,25 €)$, tres trócares $(49,22 €$ cada uno), un endoghia universal (136 €) con una carga (143,25€), una endotijera $(82,39 €)$, dos hemoclip (92,07 € cada uno), una bolsa endocath $(77,55 €)$, un irrigador aspirador $(35,57 €)$ y un ligasure $(210 €)$. El coste unitario de este material con IVA para el año 2003 nos fue suministrado por el Servicio de Compras de nuestro Centro.

Se ha utilizado el programa informático SPSS 8.0 para el manejo estadístico de las variables. Las variables cualitativas se expresan en porcentajes de las mismas y las variables cuantitativas por la media y desviación típica. Para el análisis estadístico se ha utilizado el test de Anova para las variables cuantitativas y el test de la Chi cuadrado para las cualitativas. 


\section{RESULTADOS}

No se encontraron diferencias entre ambos grupos en cuanto la edad, el sexo, el índice de masa corporal y la valoración del riesgo anestésico; en cambio, los tumores del grupo NA fueron mayores $(\mathrm{p}=0,001)$ que los del grupo NL (Tabla 1). Todas las NL fueron realizadas por un staff, mientras que el $46 \%$ de las NA fueron realizadas por un MIR.

El tiempo de utilización del quirófano, tiempo de la cirugía, coste de quirófano, coste de anestesia y de fungibles fue mayor de forma significativa en el grupo de NL. En cambio la analgesia y la estancia media fue significativamente menor en este mismo grupo (Tablas 2 y 3 ).

Tabla 1

Características de los pacientes según el grupo terapéutico.

\begin{tabular}{|c|c|c|c|}
\hline & Laparoscópica & N. Abierta & Valor de $p$ \\
\hline Pacientes & 26 & 22 & \\
\hline Edad (x \pm ds años) & $64,4 \pm 13,7$ & $60,4 \pm 13,5$ & 0,3 \\
\hline Sexo $(H / M)(\%)$ & $53 / 43$ & $47 / 57$ & 0,5 \\
\hline Riñón (D/I) (\%) & $27 / 73$ & $45,5 / 54,5$ & 0,3 \\
\hline Índice de masa corporal $(\mathrm{x} \pm \mathrm{ds})$ & $27,8 \pm 3,5$ & $26,7 \pm 2,5$ & 0,2 \\
\hline Tamaño tumoral $(\mathrm{x} \pm \mathrm{ds} \mathrm{cm})$ & $3,9 \pm 1,3$ & $5,5 \pm 1,8$ & 0,001 \\
\hline Riesgo anestésico (ASA) ( $\mathrm{x} \pm \mathrm{ds})$ & $2,1 \pm 0,5$ & $1,9 \pm 0,5$ & 0,1 \\
\hline
\end{tabular}

$\mathrm{x} \pm \mathrm{ds}=\mathrm{a}$ media \pm desviación estándar.

Tabla 2

Diferencias en tiempo según el grupo terapéutico.

\begin{tabular}{lccc}
\hline & $\begin{array}{c}\text { N. Laparoscópica } \\
\mathbf{x} \pm \mathbf{d s}\end{array}$ & $\begin{array}{c}\mathbf{N . ~ A b i e r t a} \\
\mathbf{x} \pm \mathbf{d s}\end{array}$ & Valor de p \\
\hline Tiempo de quirófano (minutos) & $295 \pm 39$ & $201 \pm 20$ & $<0,0001$ \\
Tiempo de cirugía (minutos) & $211 \pm 43$ & $138 \pm 23$ & $<0,0001$ \\
Tiempo en URPA (minutos) & $147 \pm 75$ & $185 \pm 107$ & 0,16 \\
Tiempo de UDA (días) & $0,2 \pm 0,6$ & $3 \pm 0,6$ & $<0,0001$ \\
Estancia media (días) & $3,7 \pm 1,5$ & $5,8 \pm 1,4$ & $<0,0001$ \\
\hline
\end{tabular}

URPA: Unidad de Reanimación Postoperatoria Aguda. UDA: Unidad del Dolor Agudo. $\mathrm{x} \pm \mathrm{ds}=\mathrm{a}$ media \pm desviación estándar.

Tabla 3

Diferencias de coste según el grupo terapéutico.

\begin{tabular}{lccc}
\hline & $\begin{array}{c}\text { N. Laparoscópica } \\
\mathbf{x} \pm \mathbf{d s}\end{array}$ & $\begin{array}{c}\text { N. Abierta } \\
\mathbf{x} \pm \mathbf{d s}\end{array}$ & Valor de p \\
\hline Quirófano $(€)$ & $830 \pm 112$ & $566 \pm 56$ & $<0,0001$ \\
Anestesia $(€)$ & $624 \pm 84$ & $426 \pm 42$ & $<0,0001$ \\
Fungibles $(€)$ & $1042 \pm 199$ & & \\
URPA $(€)$ & $7,2 \pm 3,8$ & $11,3 \pm 14,2$ & 0,16 \\
UDA $(€)$ & $3 \pm 10$ & $50 \pm 10$ & $<0,0001$ \\
Estancia $(€)$ & $1100 \pm 436$ & $1734 \pm 407$ & $<0,0001$ \\
Coste-Total $(€)$ & $\mathbf{3 6 0 6} \pm \mathbf{5 4 6}$ & $\mathbf{2 7 8 7 , 3} \pm \mathbf{4 0 0}$ & $<\mathbf{0 , 0 0 0 1}$ \\
\hline
\end{tabular}

URPA: Unidad de Reanimación Postoperatoria Aguda. UDA: Unidad del Dolor Agudo. $\mathrm{x} \pm \mathrm{ds}=\mathrm{a}$ media \pm desviación estándar.
La NL fue un $29,4 \%$ globalmente más cara que la NA. El coste intraoperatorio (quirófano, anestesia y fungibles) de la NL excedió en un $151,6 \%$ al de la NA, mientras que en la NA el coste postoperatorio (URPA, UDA y estancia) fue superior en un $63 \%$ con respecto a la NL.

\section{DISCUSIÓN}

La viabilidad a largo plazo de una nueva técnica terapéutica viene determinada por los beneficios para el paciente y por sus implicaciones económicas. Es muy importante la existencia de un equilibrio entre el beneficio de los pacientes y el peso económico del tratamiento. La identificación de los factores que contribuyen al coste total es el primer paso hacia la moderación de los mismos. Esto es particularmente importante en las Instituciones Públicas permanentemente preocupadas por la desviación presupuestaria.

Según Drumond ${ }^{7}$ la evaluación económica de tecnologías sanitarias conlleva un análisis comparativo entre las alternativas existentes tanto en términos de coste como de beneficio esperado. En el análisis de costes se deben de incluir los costes directos (costes de la organización y funcionamiento del sector sanitario y los sufragados por el paciente), costes indirectos (los derivados del absentismo laboral y de los cambios en la capacidad física del paciente) y los costes intangibles (relacionados con el dolor o sufrimiento del paciente).

Debido a que en un Sistema Sanitario Público como el nuestro es muy difícil evaluar los costes indirectos ya que existe suficiente literatura al respecto ${ }^{3-6}$ para considerar que la NL es tan efectiva en términos de supervivencia como la nefrectomía abierta hemos realizado un análisis de minimización de costes con el único objetivo de averiguar cuál es la opción más económica. 
Aunque las complicaciones de una técnica quirúrgica es un parámetro importante en el coste final, hemos elegido pacientes sin complicaciones ya que existe la suficiente información en la literatura para considerar que las complicaciones de la NL y la NA son equiparables ${ }^{3,4,6,8}$.

En nuestra serie la NL superó el coste total de la NA en un 29,4\%. En la serie de Meraney y Gill ${ }^{9}$ sobre 38 pacientes (20 tratados con NL y 18 con $\mathrm{NA}$ ) en las primeras NL el incremento del coste fue similar al nuestro (33\%), mientras que en las últimas la NL fue un $12 \%$ mas barata que la NA. En la serie de Lotan et al. ${ }^{10}$ sobre 19 pacientes ( 11 tratados con NL y 8 con NA) la NL fue un 16\% más barata que la NA. Lotan et al. ${ }^{11}$ compararon el coste de la NL asistida por la mano y la NA, para ello revisaron las series más recientes (últimos 5 años) recopilando un total de 546 pacientes (419 tratados mediante NL y 127 con NA) aplicándoles los costes de su Centro, la NL asistida por la mano incrementó el coste de la NA en un $3 \%$.

En nuestra serie el coste intraoperatorio de la NL fue un $151,6 \%$ superior al de la NA. En la serie de Meraney ${ }^{9}$ este incremento en el coste fue del 102\% en los casos iniciales, disminuyendo al $33 \%$ en los últimos casos. En la serie de Lotan et al. ${ }^{10}$ este incremento fue del $43 \%$. En cambio, el coste postoperatorio de la NL en nuestra serie fue un 63\% más bajo que el de la NA, cifra similar a la obtenida por Meraney ${ }^{9}$ en sus casos finales (68\%).

La implantación de una nueva técnica quirúrgica precisa de un periodo de entrenamiento hasta conseguir la eficiencia deseada. En la serie de Menarey $^{9}$ la NL en los primeros casos fue un $33 \%$ más cara que la NA, mientras que en los últimos casos la NL fue un 12\% más barata que la NA

En nuestro Centro aunque la NL consume más tiempo de quirófano, el gasto más importante son los fungibles específicos utilizados durante el procedimiento. Si pudiéramos ahorrarnos este coste tan importante (1.042 €), el coste global de la NL sería más barata que la abierta $(2.556 €$ frente a $2.754 €$ ), ya que el coste de los fungibles equivale al coste de 3,5 horas de quirófano (incluido anestesista) o de 3,5 días de estancia. Estas relaciones son completamente distin- tas en otros Sistemas Sanitarios. En la serie de Lotan et al. ${ }^{10}$ el coste de los fungibles (1.000\$) equivale al coste de la estancia de 2 días y el coste de 1 hora adicional de quirófano equivale al coste de 1,5 días de estancia.

Como ya hemos comentado en nuestro Sistema Sanitario es muy difícil evaluar los costes indirectos, a esto se une que el 54\% de los pacientes de nuestra serie eran mayores de 65 años. Según Nakada et al. ${ }^{12}$ los pacientes sometidos a NL se incorporaron a su trabajo con una media de 30,6 dias, mientras que los tratados mediante NA lo hicieron a los 53,3 días de media.

Según Novas Castro et al. ${ }^{13}$ la puesta en marcha de un programa de cirugía laparoscópica precisa de un equipo de cirujanos fijo (no más de 2), su realización en días fijos, no incorporación de nuevos miembros hasta que se haya superado la fase de aprendizaje y además, la incorporación de los miembros debe de ser paulatina y unitaria.

Este estudio adolece de varias limitaciones. Es un estudio retrospectivo. Todas las NL fueron realizadas por miembros del staff, mientras que aproximadamente la mitad (46\%) de las NA fueron llevadas a cabo por un MIR (probablemente mayor tiempo de utilización de quirófano). El tamaño de los tumores tratados mediante NA fue mayor significativamente $(3,9$ frente a $5,5 \mathrm{~cm})$, aunque creemos que esta diferencia en el tamaño es clínicamente irrelevante y por lo tanto no influyó en la dificultad técnica de la nefrectomía. Hemos excluido del análisis de coste los casos con complicaciones, aunque como ya hemos comentado según la literatura las complicaciones de ambas técnicas son equiparables.

La NL en nuestro Centro es más cara que la NA debido a un mayor tiempo de ocupación de quirófano y a que el material fungible específico utilizado en el acto quirúrgico tiene un coste muy elevado. Ya que no es probable que el material fungible específico para la NL experimente en poco tiempo una reducción importante del precio, para que la NL pudiera ser competitiva necesitaríamos conseguir una reducción en la ocupación del quirófano aproximándonos al tiempo utilizado para la NA. 


\section{REFERENCIAS}

1. Robson CJ, Churchill BM, Anderson. The results of radical nefrectomy for renal cell carcinoma. J Urol. 1969;101:297301 .

2. Clayman RV, Kavoussi LR, Soper NJ, Dierks SM, Meretyk S, Darcy MD et al. Laparoscopic nephrectomy: initial case report. J Urol. 1991;146(2):278-282.

3. Dunn MD, Portis AJ, Shalhav AL, Elbahnasy AM, Heidorn C, McDougall EM et al. Laparoscopic versus open radical neprhectomy: a 9-year experience. J. Urol. 2000;164:1153-1159.

4. Cadeddu JA, Ono Y, Clayman RV, Barrett PH, Janetschek G, Fentie DD et al. Laparoscopic neprhectomy for renal cell cancer: evaluation on efficacy and safety: a multicenter experience. Urology 1998;52(5):773-777.

5. Chan DY, Cadeddu JA, Jarrett TW, Marshall FF, Kavoussi LR. Laparoscopic radical nephrectomy: cancer control for renal cell carcinoma. J Urol. 2001;166:2095-2100.

6. Ono Y, Kinukawa T, Hattori R, Yamada S, Nishiyama N Mizutani K, et al. Laparoscopic radical nephrectomy for renal cell carcinoma: a five-year experience. Urology 1999;53:280286 .

7. Drumond M, Stoddart G, Torrance G. Methods for economic evaluation of health care programmes. Oxford: Oxford Medical Publications; 1994.

8. Gill IS, Meraney AM, Schweizer DK y col. Laparoscopic radical nephrectomy: a single institution experience of 100 cases. Cancer 2001;92:1843-1855.
9. Meraney AM, Gill IS. Financial analysis of open versus laparoscopic radical nephrectomy and nephroureterectomy. J Urol. 2002;167(4):1757-1762.

10. Lotan Y, Gettman MT, Roehrborn CG, Pearle MS, Cadeddu JA. Cost comparison for laparoscopic nephrectomy and open nephrectomy: analysis of individual parameters. Urology 2002;59(6):821-825.

11. Lotan Y, Duchene D, Cadeddu J, Koeneman KS. Cost comparison of hand assited laparoscopic nephrectomy and open nephrectomy: analysis of individual parameters. J Urol. 2003;170:752-755.

12. Nakada SY, Hedican SP, Moon TD. Hand-asited laparoscopic radical nephrectomy: update university of Wisconsin experience. J Endourol. suppl. 2002;16:A39.

13. Novas Castro S, Rubial Moldes M, González Martín M. Docencia en Urología laparoscópica. Problemas. Soluciones. Actas Urol Esp. 2005;29:241-243.

Dr. F. Herranz Amo

Lira, $4-8^{\circ} \mathrm{B}$

28007 Madrid

E-mail: felipeherranz@telefonica.net

(Trabajo recibido el 18 de abril de 2006) 